\title{
Quadratic relations between Feynman integrals
}

\author{
David Broadhurst ${ }^{* \dagger}$ \\ Open University, UK \\ E-mail: David.Broadhursteopen.ac.uk
}

David P. Roberts $\ddagger$

University of Minnesota Morris, USA

E-mail: roberts@morris.umn . edu

Feynman integrals come in two varieties: polylogarithmic, or not. They are used in two ways: as contributions to an amplitude that is squared, or as contributions to an observable matrix element. In the former case, products of integrals occur, in the latter they do not. We report on products of non-polylogarithmic Feynman integrals related to the magnetic moment of the electron, giving details of an infinite set of quadratic relations between these integrals at all loops $L>2$.

Loops and Legs in Quantum Field Theory (LL2018)

29 April 2018 - 04 May 2018

St. Goar, Germany

\footnotetext{
* Speaker.

${ }^{\dagger}$ DB thanks the organizers of LL2018 for financial support.

${ }^{\ddagger}$ DPR’s research is supported by grant DMS-1601350 from the National Science Foundation.
} 


\section{Four-loop sunrise: the electron's magnetic moment}

As reported in [9], the magnetic moment of the electron in Bohr magnetons has QED contributions $\sum_{L \geq 0} a_{L}(\alpha / \pi)^{L}$ given up to $L=4$ loops by

$$
\begin{aligned}
& a_{0}=1 \\
& a_{1}=0.5 \\
& a_{2}=-0.32847896557919378458217281696489239241111929867962 \ldots \\
& a_{3}=1.18124145658720000627475398221287785336878939093213 \ldots \\
& a_{4}=-1.91224576492644557415264716743983005406087339065872 \ldots
\end{aligned}
$$

with a trilogarithm in

$$
a_{2}=\frac{197}{144}+\frac{\zeta(2)}{2}+\frac{3 \zeta(3)-2 \pi^{2} \log 2}{4}
$$

and a weight 4 depth 2 polylogarithm $U_{3,1}:=\sum_{m>n>0}(-1)^{m+n} /\left(m^{3} n\right)$ in

$$
\begin{aligned}
a_{3}= & \frac{28259}{5184}+\frac{17101 \zeta(2)}{135}+\frac{139 \zeta(3)-596 \pi^{2} \log 2}{18} \\
& -\frac{39 \zeta(4)+400 U_{3,1}}{24}-\frac{215 \zeta(5)-166 \zeta(3) \zeta(2)}{24}
\end{aligned}
$$

\subsection{The first non-polylogarithm}

At 4 loops, a Bessel moment

$$
\begin{aligned}
B & =-\int_{0}^{\infty} \frac{27550138 x+35725423 x^{3}}{48600} I_{0}(x) K_{0}^{5}(x) \mathrm{d} x \\
& =-1483.68505914882529459059985184510836700500152630607810 \ldots
\end{aligned}
$$

occurs at weight 4 in the breathtaking evaluation by Stefano Laporta [9] of 4800 digits of

$$
a_{4}=P+B+E+U \approx 2650.565-1483.685-1036.765-132.027 \approx-1.912
$$

where $P$ comprises polylogs and $E$ comprises integrals, with weights 5, 6 and 7, whose integrands contain logs and products of elliptic integrals. $U$ comes from 6 light-by-light master integrals, still under investigation.

The weight- 4 non-polylog term $B$ has $N=6$ Bessel functions, with 5 instances of $K_{0}(x)$, from 5-fermion intermediate states. The sibling of $K_{0}(x)$ is $I_{0}(x)=\sum_{k \geq 0}\left((x / 2)^{k} / k !\right)^{2}$, from Fourier transformation. Both master integrals in $B$ occur in $D=2$ spacetime dimensions.

\subsection{A simple determinant of Bessel moments}

Consider Bessel moments of the form [1]

$$
M(a, b, c):=\int_{0}^{\infty} I_{0}^{a}(x) K_{0}^{b}(x) x^{c} \mathrm{~d} x
$$


The moment $2^{L} M(1, L+1,1)$ is the $L$-loop sunrise integral at $D=2$, on shell:

$$
\begin{aligned}
& S_{L}(t):=\int_{0}^{\infty} \frac{\mathrm{d} x_{1}}{x_{1}} \ldots \int_{0}^{\infty} \frac{\mathrm{d} x_{L}}{x_{L}} \frac{1}{\left(1+\sum_{j=1}^{L} x_{j}\right)\left(1+\sum_{k=1}^{L} 1 / x_{k}\right)-t} \\
& S_{4}(1)=2^{4} M(1,5,1):=2^{4} \int_{0}^{\infty} I_{0}(x) K_{0}^{5}(x) x \mathrm{~d} x .
\end{aligned}
$$

Laporta encountered $M(1,5,1)$ as a master integral at $D=4$. He also encountered $M(1,5,3)$, which is obtained by differentiation of $S_{4}(t)$ before setting $t=1$. Now look at the determinant $[4,12]$

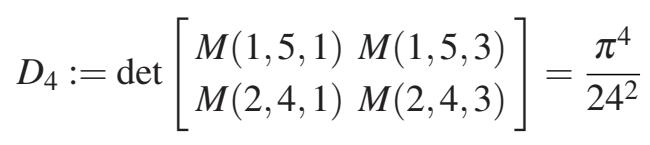

where $M(2,4,1)$ comes from cutting an internal line. It occurred at stages of Laporta's $\varepsilon$-expansions. $M(2,4,3)$ comes from a cut and differentiation.

\section{Simple determinants up to $L=6$ loops}

At $L$ loops, with $N=L+2$ Bessel functions, there is a simple result for a $k \times k$ determinant with $k=\lfloor(L+1) / 2\rfloor$. The first non-trivial case is at $L=3$ loops, where it was discovered [3] and is now proven [14] that

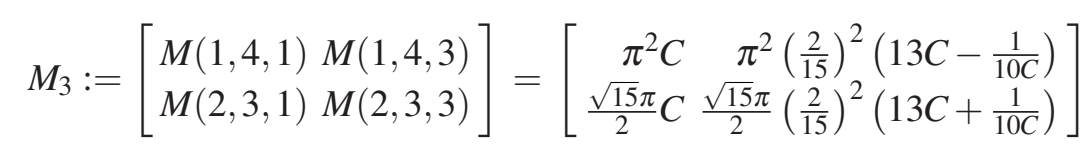

is determined by

$$
C=\frac{\Gamma\left(\frac{1}{15}\right) \Gamma\left(\frac{2}{15}\right) \Gamma\left(\frac{4}{15}\right) \Gamma\left(\frac{8}{15}\right)}{240 \sqrt{5} \pi^{2}}, \quad \frac{1}{C}=\frac{75 \Gamma\left(\frac{7}{15}\right) \Gamma\left(\frac{11}{15}\right) \Gamma\left(\frac{13}{15}\right) \Gamma\left(\frac{14}{15}\right)}{\sqrt{5} \pi^{2}}
$$

which ensure a simple determinant at 3 loops $[4,12]$

$$
D_{3}:=\operatorname{det} M_{3}=2 \pi^{3} / \sqrt{3^{3} 5^{5}}
$$

\subsection{Hypergeometric identity at 4 loops}

For the Laporta problem, the Feynman integrals are combinations of [13]

$$
\begin{array}{lr}
F_{a}={ }_{4} F_{3}(1 / 2,2 / 3,2 / 3,5 / 6 ; & 7 / 6,7 / 6,4 / 3 ; 1) \\
F_{b}={ }_{4} F_{3}(-1 / 2,1 / 6,1 / 3,4 / 3 ; & -1 / 6,5 / 6,5 / 3 ; 1) \\
F_{c}={ }_{4} F_{3}(1 / 6,1 / 3,1 / 3,1 / 2 ; & 2 / 3,5 / 6,5 / 6 ; 1) \\
F_{d}={ }_{4} F_{3}(-7 / 6,-1 / 2,-1 / 3,2 / 3 ; & -5 / 6,1 / 6,1 / 3 ; 1)
\end{array}
$$

with a quadratic relation $7 F_{a} F_{b}+10 F_{c} F_{d}=40$ giving $D_{4}=\pi^{4} / 24^{2}$ in (1.15).

\subsection{Hidden quadratic relations at 5 loops}

The corresponding determinant $[4,12] D_{5}=16 \pi^{6} / \sqrt{3^{3} 5^{5} 7^{7}}$ involves products of three Feynman integrals. We shall show that this results from a substructure of several quadratic relations. 


\subsection{Quadratic relation at 6 loops}

The correspondng $3 \times 3$ determinant $[4,12] D_{6}=5 \pi^{8} /\left(2^{19} 3\right)$ comes from a quadratic relation

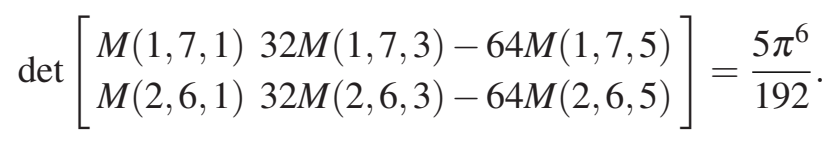

\section{Feynman integrals from modular forms}

Until recently, relations between Feynman integrals and Eichler integrals of modular forms were conjectural $[2,3,6,4]$, tested to many thousands of digits. For an account of how they were proved [10, 11, 12, 13], see the lucid review by Yajun Zhou [14].

With $q:=\exp (2 \pi \mathrm{i} z)$ and $\mathfrak{I} z>0$, the Dedekind eta function satisfies

$$
\eta(z):=q^{1 / 24} \prod_{n=1}^{\infty}\left(1-q^{n}\right)=\sum_{n=-\infty}^{\infty}(-1)^{n} q^{(6 n+1)^{2} / 24}=\frac{\eta(-1 / z)}{\sqrt{-\mathrm{i} z}} .
$$

With $\eta_{n}:=\eta(n z)$, we define the weight-3 level-15 cuspform

$$
f_{3,15}(z):=\left(\eta_{3} \eta_{5}\right)^{3}+\left(\eta_{1} \eta_{15}\right)^{3}=\sum_{n>0} A_{5}(n) q^{n}=-\frac{f_{3,15}(-1 /(15 z))}{(-15)^{3 / 2} z^{3}} .
$$

If the Kronecker symbol $\left(\frac{p}{15}\right)=\left(\frac{p}{3}\right)\left(\frac{p}{5}\right)$ is negative, for prime $p$, then $A_{5}(p)=0$. For $\Re s>2$, there is a convergent $\mathrm{L}$-series

$$
L_{5}(s)=\sum_{n>0} \frac{A_{5}(n)}{n^{s}}=\prod_{p} \frac{1}{1-A_{5}(p) p^{-s}+\left(\frac{p}{15}\right) p^{2-2 s}} .
$$

Its analytic continuation is provided by the Eichler integral

$$
L_{5}(s)=\frac{(2 \pi)^{s}}{\Gamma(s)} \int_{0}^{\infty} f_{3,15}(\mathrm{i} y) y^{s-1} \mathrm{~d} y
$$

with critical values

$$
L_{5}(1)=\frac{5}{\pi^{2}} \int_{0}^{\infty} I_{0}(x) K_{0}^{4}(x) x \mathrm{~d} x, \quad L_{5}(2)=\frac{4}{3} \int_{0}^{\infty} I_{0}^{2}(x) K_{0}^{3}(x) x \mathrm{~d} x .
$$

\subsection{A modular L-series at 4 loops}

Consider the Fourier expansion of the weight- 4 level- 6 cuspform

$$
f_{4,6}(z):=\left(\eta_{1} \eta_{2} \eta_{3} \eta_{6}\right)^{2}=\sum_{n>0} A_{6}(n) q^{n}=\frac{f_{4,6}(-1 /(6 z))}{6^{2} z^{4}} .
$$

For $\Re_{S}>5 / 2$, there is a convergent L-series

$$
L_{6}(s)=\sum_{n>0} \frac{A_{6}(n)}{n^{s}}=\frac{1}{1+2^{1-s}} \frac{1}{1+3^{1-s}} \prod_{p>3} \frac{1}{1-A_{6}(p) p^{-s}+p^{3-2 s}} .
$$


Its analytic continuation is provided by the Eichler integral

$$
L_{6}(s)=\frac{(2 \pi)^{s}}{\Gamma(s)} \int_{0}^{\infty} f_{4,6}(\mathrm{i} y) y^{s-1} \mathrm{~d} y
$$

with critical values related to Bessel moments as follows

$$
\begin{aligned}
& L_{6}(2)=\frac{2}{\pi^{2}} M(1,5,1)=\frac{2}{3} M(3,3,1), \\
& L_{6}(1)=\frac{2}{\pi^{2}} M(2,4,1)=\frac{3}{\pi^{2}} L_{6}(3) .
\end{aligned}
$$

\subsection{A non-modular L-series at 5 loops}

With 7 Bessel functions and $\Re s>3$, the local factors at the primes in

$$
L_{7}(s)=\prod_{p} \frac{1}{Z_{7}\left(p, p^{-s}\right)}
$$

are given, for $p$ coprime to 105 , by the cubic

$$
Z_{7}(p, T)=\left(1-\left(\frac{p}{105}\right) p^{2} T\right)\left(1+\left(\frac{p}{105}\right)\left(2 p^{2}-\left|\lambda_{p}\right|^{2}\right) T+p^{4} T^{2}\right)
$$

where $\lambda_{p}$ is a complex Hecke eigenvalue of a weight-3 newform with level 525. For $p \mid 105$, one obtains, from Kloosterman moments [3] in finite fields,

$$
Z_{7}(3, T)=1-10 T+3^{4} T^{2}, Z_{7}(5, T)=1-5^{4} T^{2}, Z_{7}(7, T)=1+70 T+7^{4} T^{2},
$$

which enable discovery of the functional equation [4]

$$
\Lambda_{7}(s):=\left(\frac{105}{\pi^{3}}\right)^{s / 2} \Gamma\left(\frac{s-1}{2}\right) \Gamma\left(\frac{s}{2}\right) \Gamma\left(\frac{s+1}{2}\right) L_{7}(s)=\Lambda_{7}(5-s) .
$$

Then Tim Dokchitser's package COMPUTEL [8] gives the empirical result

$$
L_{7}(2)=\frac{24}{5 \pi^{2}} \int_{0}^{\infty} I_{0}^{2}(x) K_{0}^{5}(x) x \mathrm{~d} x .
$$

\subsection{A modular L-series at 6 loops}

Consider the Fourier expansion of the weight- 6 level- 6 cuspform

$$
f_{6,6}(z):=\frac{\eta_{2}^{9} \eta_{3}^{9}}{\eta_{1}^{3} \eta_{6}^{3}}+\frac{\eta_{1}^{9} \eta_{6}^{9}}{\eta_{2}^{3} \eta_{3}^{3}}=\sum_{n>0} A_{8}(n) q^{n}=-\frac{f_{6,6}(-1 /(6 z))}{6^{3} z^{6}} .
$$

For $\Re s>7 / 2$, there is a convergent L-series

$$
L_{8}(s)=\sum_{n>0} \frac{A_{8}(n)}{n^{s}}=\frac{1}{1-2^{2-s}} \frac{1}{1+3^{2-s}} \prod_{p>3} \frac{1}{1-A_{8}(p) p^{-s}+p^{5-2 s}} .
$$

Its analytic continuation is provided by the Eichler integral

$$
L_{8}(s)=\frac{(2 \pi)^{s}}{\Gamma(s)} \int_{0}^{\infty} f_{6,6}(\mathrm{i} y) y^{s-1} \mathrm{~d} y
$$

with critical values related to Bessel moments as follows

$$
\begin{aligned}
& L_{8}(4)=\frac{4}{9 \pi^{2}} M(1,7,1)=\frac{4}{9} M(3,5,1)=\frac{\pi^{2}}{9} L_{8}(2), \\
& L_{8}(5)=\frac{4}{27} M(2,6,1)=\frac{2 \pi^{2}}{21} M(4,4,1)=\frac{2 \pi^{2}}{21} L_{8}(3)=\frac{\pi^{4}}{54} L_{8}(1) .
\end{aligned}
$$




\section{Quasi-periods associated to modular forms}

In [7] Francis Brown associated a pair of periods and a pair of quasi-periods to the weight 12 level 1 modular form $\Delta(z)=\eta_{1}^{24}$. The periods are a pair of Eichler integrals that determine critical values of the L-series at odd and even integers. No concrete integrals were given for the quasi-periods. Rather it was asserted that numerical values may be obtained by an undeclared regularization of integrals of a weakly holomorphic modular form $\Delta^{\prime}(z)=1 / q+O\left(q^{2}\right)$.

\subsection{Quasi-periods from 4-loop sunrise}

In terms of Eichler integrals,

$$
\begin{aligned}
\frac{D_{2}}{2}=\frac{M(1,5,1)}{\pi^{4}} & =\frac{4 M(1,5,3)}{\pi^{4}}+\frac{5 E_{2}}{18}, \\
\frac{3 D_{1}}{5}=\frac{M(2,4,1)}{\pi^{3}} & =\frac{4 M(2,4,3)}{\pi^{3}}+\frac{E_{1}}{3}, \\
{\left[\begin{array}{c}
D_{s} \\
E_{s}
\end{array}\right] } & :=-\int_{1 / \sqrt{3}}^{\infty}\left[\begin{array}{l}
f_{4,6}\left(\frac{1+\mathrm{i} y}{2}\right) \\
g_{4,6}\left(\frac{1+\mathrm{i} y}{2}\right)
\end{array}\right] y^{s-1} \mathrm{~d} y, \\
g_{4,6}(z):=\frac{\left(w^{2}-3\right)^{2}\left(w^{4}+9\right)}{8 w^{4}} f_{4,6}(z) & =5 q+102 q^{2}+945 q^{3}+O\left(q^{4}\right), \\
w & :=3 \frac{\eta_{2}^{2} \eta_{3}^{4}}{\eta_{1}^{4} \eta_{6}^{2}}, \\
D_{1} E_{2}-D_{2} E_{1} & =\frac{1}{24 \pi^{3}},
\end{aligned}
$$

with a simple determinant relating a pair of periods and a pair of quasi-periods.

\subsection{Quasi-periods from six-loop sunrise}

We found empirical relations to Eichler integrals for the second column of

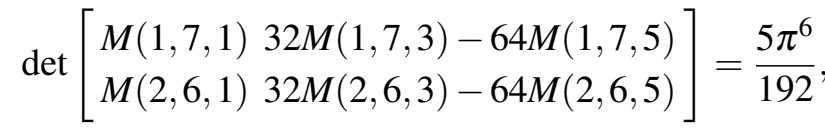

$$
\begin{aligned}
& \frac{F_{2}}{4}=\frac{M(1,7,1)}{\pi^{6}}=\frac{32 M(1,7,3)-64 M(1,7,5)}{\pi^{6}}+\frac{35 G_{2}}{108}, \\
& \frac{9 F_{1}}{28}=\frac{M(2,6,1)}{\pi^{5}}=\frac{32 M(2,6,3)-64 M(2,6,5)}{\pi^{5}}+\frac{5 G_{1}}{12}, \\
& {\left[\begin{array}{l}
F_{s} \\
G_{s}
\end{array}\right]:=-\int_{1 / \sqrt{3}}^{\infty}\left[\begin{array}{l}
f_{6,6}\left(\frac{1+\mathrm{i} y}{2}\right) \\
g_{6,6}\left(\frac{1+\mathrm{i} y}{2}\right)
\end{array}\right]\left(3 y^{2}-1\right) y^{s-1} \mathrm{~d} y,} \\
& g_{6,6}(z):=\frac{\left(w^{2}-3\right)^{4}}{16 w^{4}} f_{6,6}(z)=q+36 q^{2}+567 q^{3}+5264 q^{4}+O\left(q^{5}\right) \text {, } \\
& F_{1} G_{2}-F_{2} G_{1}=\frac{1}{4 \pi^{5}},
\end{aligned}
$$

with $\left(3 y^{2}-1\right)$ inferred from the dispersion relation for a sub-diagram. Note that the integrand of $G_{s}$ is of order $\left(3 y^{2}-1\right)^{6}$ near its threshold. A link to Francis Brown's concept of quasi-periods is forming, yet is not complete, since $g_{6,6}$ lacks a period polynomial enjoyed by $f_{6,6}$. 


\subsection{Quasi-periods from even Bessel moments}

At weight 4 and level 8, we obtained the empirical relations

$$
\begin{aligned}
{\left[\begin{array}{c}
2 M(0,4,0) 4 M(0,4,0)-16 M(0,4,2) \\
2 M(1,3,0) 4 M(1,3,0)-16 M(1,3,2)
\end{array}\right] } & =\left[\begin{array}{ll}
\pi^{4} P_{1} & 3 \pi^{4} Q_{1} \\
\pi^{3} P_{2} & 3 \pi^{3} Q_{2}
\end{array}\right] \\
{\left[\begin{array}{c}
P_{s} \\
Q_{s}
\end{array}\right] } & :=-\mathrm{i} \int_{1}^{\infty}\left[\begin{array}{c}
f_{4,8}\left(\frac{1+\mathrm{i} y}{4}\right) \\
g_{4,8}\left(\frac{1+\mathrm{i} y}{4}\right)
\end{array}\right] \frac{y^{s}+y^{4-s}}{y} \mathrm{~d} y, \\
f_{4,8}(z):=\left(\eta_{2} \eta_{4}\right)^{4} & =q-4 q^{3}-2 q^{5}+O\left(q^{7}\right) \\
g_{4,8}(z):=\left(1+64 \frac{\eta_{4}^{24}}{\eta_{2}^{24}}\right) f_{4,8}(z) & =q+60 q^{3}+1278 q^{5}+O\left(q^{7}\right) \\
P_{1} Q_{2}-P_{2} Q_{1} & =-\frac{1}{2 \pi^{3}}
\end{aligned}
$$

with $g_{4,8}\left(z_{0}\right)=0$ at $z_{0}=(1+\mathrm{i}) / 4$, where $-\mathrm{i} f_{4,8}\left(z_{0}\right)=\Gamma^{8}(1 / 4) /\left(128 \pi^{6}\right)$.

\subsection{Periods at level 24}

The unique weight- 6 Hecke eigenform that is both a newform of level 24 and also has a negative sign in the functional equation for its L-series is

$$
\begin{aligned}
f_{6,24}(z):= & \frac{\eta_{3}^{4} \eta_{4}^{2} \eta_{6}^{6} \eta_{8}^{2}}{\eta_{24}^{2}}+\frac{\eta_{1}^{4} \eta_{2}^{6} \eta_{12}^{2} \eta_{24}^{2}}{3 \eta_{8}^{2}}-\frac{16 \eta_{1}^{2} \eta_{2}^{2} \eta_{12}^{6} \eta_{24}^{4}}{\eta_{3}^{2}}-\frac{16 \eta_{3}^{2} \eta_{4}^{6} \eta_{6}^{2} \eta_{8}^{4}}{3 \eta_{1}^{2}} \\
& +\frac{64 \eta_{1}^{2} \eta_{3}^{2} \eta_{4} \eta_{8}^{4} \eta_{12} \eta_{24}^{4}}{\eta_{2} \eta_{6}}-\frac{4 \eta_{1}^{4} \eta_{2} \eta_{3}^{4} \eta_{6} \eta_{8}^{2} \eta_{24}^{2}}{\eta_{4} \eta_{12}}=-f_{6,24}(z+1 / 2) \\
= & \frac{f_{6,24}(-1 /(24 z))}{24^{3} z^{6}}=-\frac{f_{6,24}((3 z-1) /(12 z-3))}{3^{3}(4 z-1)^{6}} \\
= & q-9 q^{3}-34 q^{5}-240 q^{7}+81 q^{9}-124 q^{11}+46 q^{13}+O\left(q^{15}\right) .
\end{aligned}
$$

We empirically related its critical L-series to Bessel moments:

$$
\widetilde{L}_{6}(4)=\frac{M(0,6,0)}{108 \pi^{2}}, \quad \widetilde{L}_{6}(5)=\frac{M(1,5,0)}{144}, \quad \widetilde{L}_{6}(s):=\frac{(2 \pi)^{s}}{\Gamma(s)} \int_{0}^{\infty} f_{6,24}(\mathrm{i} y) y^{s-1} \mathrm{~d} y .
$$

\subsection{Striving for quasi-periods at level 24}

After intensive experiment at high precision, we conjecture that

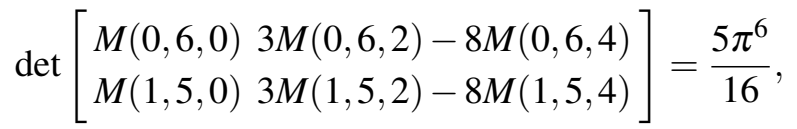

$$
\begin{aligned}
& \frac{M(0,6,0)}{\pi^{6}}=\frac{R_{1}}{28}=\frac{3 R_{3}}{4}, \quad \frac{M(1,5,0)}{\pi^{5}}=\frac{R_{2}}{8}, \\
& R_{s}:=-\mathrm{i} \int_{0}^{\infty} f_{6,24}\left(\frac{1+\mathrm{i} y}{4}\right) y^{s-1} \mathrm{~d} y=3^{3-s} R_{6-s} .
\end{aligned}
$$

So far we have not succeeded in relating the second column of the determinant to Eichler integrals of a weakly holomorphic modular form. 


\section{Quadratic relations between integrals for all loops}

Conjecture: With the Feynman, de Rham and Betti matrices below, we conjecture that

$$
F_{N} D_{N} F_{N}^{\mathrm{tr}}=B_{N}
$$

The elements of $F_{N}$ are given as Bessel moments by

$$
\begin{aligned}
& F_{2 k+1}(u, a):=\frac{(-1)^{a-1}}{\pi^{u}} M(k+1-u, k+u, 2 a-1) \\
& F_{2 k+2}(u, a):=\frac{(-1)^{a-1}}{\pi^{u+1 / 2}} M(k+1-u, k+1+u, 2 a-1)
\end{aligned}
$$

with $u$ and $a$, as well as later indices $v$ and $b$, running from 1 to $k$. In (5.1), $F_{N}^{\text {tr }}$ is the transpose of the Feynman matrix $F_{N}$. The Betti matrices $B_{N}$ have rational elements given by

$$
\begin{aligned}
B_{2 k+1}(u, v) & :=(-1)^{u+k} 2^{-2 k-2}(k+u) !(k+v) ! Z(u+v) \\
B_{2 k+2}(u, v) & :=(-1)^{u+k} 2^{-2 k-3}(k+u+1) !(k+v+1) ! Z(u+v+1) \\
Z(m) & =\frac{\left|\mathscr{B}_{m}\right|}{m !},
\end{aligned}
$$

with the absolute value of the $m$-th Bernoulli number appearing in (5.6). Our original construction of the rational de Rham matrices $D_{N}$ at the Matrix Institute [5] was very intricate. We have more recently substantially simplified it as follows. Let $v_{k}$ and $w_{k}$ be the rational numbers generated by

$$
\begin{aligned}
\frac{J_{0}^{2}(t)}{C(t)} & =\sum_{k \geq 0} \frac{v_{k}}{k !}\left(\frac{t}{2}\right)^{2 k}=1-\frac{17 t^{2}}{54}+\frac{3781 t^{4}}{186624}+\ldots \\
\frac{2 J_{0}(t) J_{1}(t)}{t C(t)} & =\sum_{k \geq 0} \frac{w_{k}}{k !}\left(\frac{t}{2}\right)^{2 k}=1-\frac{41 t^{2}}{216}+\frac{325 t^{4}}{186624}+\ldots
\end{aligned}
$$

where $J_{0}(t)=I_{0}(\mathrm{i} t), J_{1}(t)=-J_{0}^{\prime}(t)$ and

$$
C(t):=\frac{32\left(1-J_{0}^{2}(t)-t J_{0}(t) J_{1}(t)\right)}{3 t^{4}}=1-\frac{5 t^{2}}{27}+\frac{35 t^{4}}{2304}-\frac{7 t^{6}}{9600}+\ldots
$$

Construct rational bivariate polynomials $H_{s}=H_{s}(y, z)$ by the recursion

$$
H_{s}=z H_{s-1}-(s-1) y H_{s-2}-\sum_{k=1}^{s-1}\left(\begin{array}{c}
s-1 \\
k
\end{array}\right)\left(v_{k} H_{s-k}-w_{k} z H_{s-k-1}\right)
$$

for $s>0$, with $H_{0}=1$ and $H_{-1}=0$. Use these to define

$$
d_{s}(N, c):=\frac{H_{s}(3 c / 2, N+2-2 c)}{4^{s} s !} .
$$

Finally, construct rational de Rham matrices, with elements

$$
D_{N}(a, b):=\sum_{c=-b}^{a} d_{a-c}(N,-c) d_{b+c}(N, c) c^{N+1} .
$$




\section{Summary}

We have shown that Laporta's four-loop result [9] contains a modular quasi-period in $(4.1,4.6)$. Simple determinants occur for all loops $L>2$, though the corresponding L-series are modular only for $L=3,4$ and 6 loops. At 6 loops we encountered quasi-periods in (4.10,4.12). For all loops $L>2$, with $N=L+2$ Bessel functions, there are quadratic relations of the form $F_{N} D_{N} F_{N}^{\mathrm{tr}}=B_{N}$ with Feynman, de Rham and Betti matrices that have been specified.

\section{References}

[1] David H. Bailey, Jonathan M. Borwein, David Broadhurst and M.L. Glasser, Elliptic integral evaluations of Bessel moments, J. Phys. A 41 (2008) 205203 [arXiv:0801.0891].

[2] David Broadhurst, Multiple zeta values and modular forms in quantum field theory, in Computer Algebra in Quantum Field Theory (ed. C. Schneider and J. Blümlein), Texts \& Monographs in Symbolic Computation, 33-73, Springer-Verlag, Vienna, 2013.

[3] David Broadhurst, Feynman integrals, L-series and Kloosterman moments, Commun. Number Theory Phys. 10 (2016) 527-569 [arXiv:1604.03057].

[4] David Broadhurst and Anton Mellit, Perturbative quantum field theory informs algebraic geometry, in Loops and Legs in Quantum Field Theory, PoS (LL2016) 079, 2016.

[5] David Broadhurst and David P. Roberts, L-series and Feynman integrals, 2017 MATRIX Annals, Editors: David R. Wood, Jan de Gier, Cheryl E. Praeger, Terence Tao. MATRIX Book Series, Volume 2, Springer, to appear, http: //www.matrix-inst.org.au/2017-matrix-annals/.

[6] David Broadhurst and Oliver Schnetz, Algebraic geometry informs perturbative quantum field theory, in Loops and Legs in Quantum Field Theory, PoS (LL2014) 078, 2014.

[7] Francis Brown, A class of non-holomorphic modular forms III: real analytic cusp forms for $S L_{2}(Z)$ [arXiv:1710.07912].

[8] Tim Dokchitser, Computing special values of motivic L-functions, Experimental Mathematics 13 (2004) 137-149 [arXiv:math/0207280].

[9] Stefano Laporta, High-precision calculation of the 4-loop contribution to the electron $g-2$ in QED, Phys. Lett. B 772 (2017) 232-238 [arXiv:1704.06996].

[10] Yajun Zhou, Hilbert transforms and sum rules of Bessel moments, Ramanujan J. (2017) http://doi.org/10.1007/s11139-017-9945-y [arXiv:1706.01068].

[11] Yajun Zhou, Wick rotations, Eichler integrals, and multi-loop Feynman diagrams, Commun. Number Theory Phys. 12 (2018) 127-192, [arXiv:1706.08308].

[12] Yajun Zhou, Wronskian factorizations and Broadhurst-Mellit determinant formulae, Commun. Number Theory Phys. 12 (2018) 355-407 [arXiv:1711.01829].

[13] Yajun Zhou, On Laporta's 4-loop sunrise formulae [arXiv:1801.02182].

[14] Yajun Zhou, Some algebraic and arithmetic properties of Feynman diagrams [arXiv:1801.05555]. 